\title{
Avaliação do peso de estudantes durante o isolamento social da pandemia COVID-19
}

\author{
Assessment of student weight during the social isolation of the COVID-19 pandemic \\ Evaluación del peso de los estudiantes durante el aislamiento social de la pandemia COVID-19
}

Recebido: 03/02/2022 | Revisado: 08/02/2022 | Aceito: 12/02/2022 | Publicado: 17/02/2022

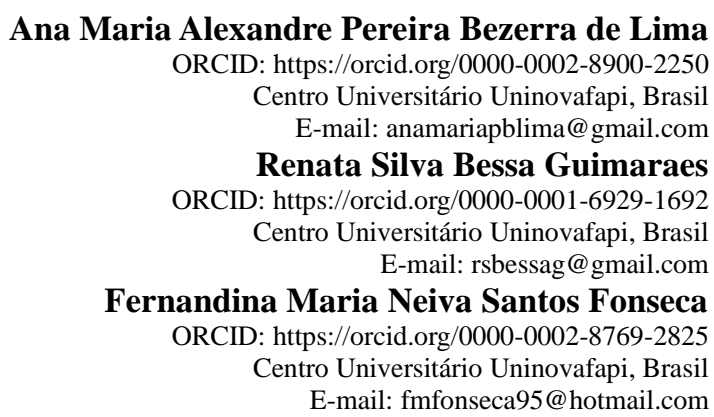

\begin{abstract}
Resumo
Este estudo tem como objetivo verificar a variação ponderal de estudantes do Ensino Médio de uma escola particular de Teresina-Piauí durante o isolamento social devido à pandemia COVID-19. Tratou-se de um estudo epidemiológico observacional, por meio de coleta de XX formulários de estudantes, pelo qual foi realizado um levantamento dos dados sócio-demográficos. Com os resultados deste estudo, observou-se que os dados da pesquisa sugerem que a pandemia ocasionada pelo Sars-Cov-2 foi associado a maiores aumentos no peso corporal depois do que antes da pandemia. A disseminação da regulação alimentar e de estratégias de enfrentamento saudáveis é de importância crítica durante a pandemia de COVID-19 para prevenir o ganho de peso e padrões alimentares pouco saudáveis.
\end{abstract}

Palavras-chave: COVID-19; Isolamento social; Alterações de peso.

\begin{abstract}
This study aims to verify the weight variation of high school students from a private school in Teresina-Piauí during social isolation due to the COVID-19 pandemic. This was an observational epidemiological study, through the collection of XX student forms, through which a survey of socio-demographic data was carried out. With the results of this study, it was observed that Research data suggest that the pandemic caused by Sars-Cov-2 was associated with greater increases in body weight after the pandemic than before. The spread of dietary regulation and healthy coping strategies is of critical importance during the COVID-19 pandemic to prevent weight gain and unhealthy eating patterns.
\end{abstract}

Keywords: COVID-19; Social isolation; Body weigh.

\section{Resumen}

Este estudio tiene como objetivo verificar la variación de peso de los estudiantes de secundaria de una escuela privada en Teresina-Piauí durante el aislamiento social debido a la pandemia de COVID-19. Se trató de un estudio epidemiológico observacional, mediante la recolección de XX formularios de estudiantes, mediante el cual se realizó una encuesta de datos sociodemográficos. Con los resultados de este estudio, se observó que los datos de la investigación sugieren que la pandemia causada por Sars-Cov-2 se asoció con mayores incrementos en el peso corporal después de la pandemia que antes. La difusión de la regulación dietética y las estrategias de afrontamiento saludables es de importancia crítica durante la pandemia de COVID-19 para prevenir el aumento de peso y los patrones de alimentación poco saludables.

Palabras clave: COVID-19; Aislamiento social; Cambios em el peso.

\section{Introdução}

A avaliação do estado nutricional tem se tornado aspecto cada vez mais importante no estabelecimento de situações de risco, no diagnóstico nutricional e no planejamento de ações de promoção à saúde e prevenção de doenças. Sua importância é reconhecida tanto na atenção primária, para acompanhar o crescimento e a saúde da criança e do adolescente, quanto na detecção precoce de distúrbios nutricionais, seja desnutrição, seja obesidade (SBP, 2009).

O novo coronavírus, causador da COVID-19, espalhou-se pelo mundo desde dezembro de 2019. Com o aumento do 
número de pessoas infectadas e de óbitos causados por esta infecção, é considerado uma pandemia pela Organização Mundial de Saúde. Assim, devido a tais proporções, a quarenta e o isolamento social tem sido utilizado como principal estratégia de contenção (Rodrigues; Lins, 2020).

Para Su et al. (2016), na década de 60, os coronavírus humanos (HCoVs) foram descritos pela primeira vez em pacientes com resfriado comum. Desde então, mais HCoVs foram descobertos, incluindo os que evoluem para síndrome respiratória aguda (SARS) e para síndrome respiratória do Oriente Médio (MERS), ambos os casos podem evoluir para um desfecho fatal em humanos.

Segundo Zhu et al. (2020), em dezembro de 2019, pacientes com pneumonia viral por um patógeno desconhecido foram reportados em Wuhan, China. O fator epidemiológico comum associado seria o acesso ao mercado local de Huanan, onde havia grande quantidade de animais, como coelhos e pássaros, à venda antes do surto. Um novo coronavírus foi identificado, provisoriamente chamado de novo coronavírus 2019 (2019-nCoV), nas amostras do lavado broncoalveolar de 9 pacientes, dos quais 8 teriam visitado o local supracitado.

Chan et al. (2020) sugere que a transmissão pessoa-para-pessoa e a transmissão interestadual do novo coronavírus por viagens aéreas são possíveis, de acordo com os casos de viajantes chineses de Wuhan sendo detectados em regiões demográficas divergentes. Logo, medidas de vigilância devem ser tomadas para o controle da epidemia, sendo crucial o isolamento dos pacientes aliado à quarentena dos indivíduos contactantes o mais breve possível, pois a infecção pode ser assintomática. Ademais, deve-se educar o público, inclusive profissionais de saúde, sobre a higienização correta dos alimentos e higiene pessoal para prevenir eventos de super-pulverização do vírus.

Em 30 de janeiro de 2020, a Organização Mundial da Saúde (OMS) declarou a epidemia de COVID-19 'emergência de importância internacional', e, em 11 de março de 2020, foi declarada a pandemia (Szwarcwald et al 2020). Em 5 de abril de 2020, segundo Qiu, Chen e Shi (2020), mais de 1,2 milhões de casos confirmados foram reportados em pelo menos duzentos países ou territórios e mais de 64,7 mil óbitos registrados. O primeiro caso de COVID-19 no Brasil foi identificado em 26 de fevereiro e, até o final de maio, haviam sido registrados 514.200 casos e 29.310 mortes no país (Szwarcwald et al 2020).

Até a descoberta de intervenções médicas, medidas para redução de $90 \%$ de contato social e laboral, além de familiar, aliado ao fechamento de escolas foram tomadas. Para evitar a sobrecarga dos sistemas de saúde e retardar a disseminação da COVID-19, vários países têm implementado medidas de restrição ao contato físico ou social, desde o fechamento de escolas e estabelecimentos comerciais até quarentenas de alcance nacional. Até maio de 2020, grande parte da população mundial se encontrava sob alguma forma de restrição, com o propósito de se evitar a aglomeração de pessoas (Szwarcwald et al., 2020).

No Brasil, diversas medidas foram adotadas pelos estados e municípios, como o fechamento de escolas e comércios não essenciais. Trabalhadores foram orientados a desenvolver suas atividades em casa, alguns municípios e estados encerraram-se em seus limites e divisas. Autoridades públicas locais chegaram a decretar bloqueio total (lockdown), com punições para estabelecimentos e indivíduos que não se adequassem às normativas. A restrição social resulta ser a medida mais difundida pelas autoridades, e a mais efetiva para evitar a disseminação da doença e achatar a curva de transmissão do coronavírus. Geralmente, a repercussão clínica e comportamental dessa obrigação implica mudanças no estilo de vida e pode afetar a saúde mental dos cidadãos (Malta et al., 2020).

Em relação aos estilos de vida, a restrição social pode levar a uma redução importante nos níveis de atividade física de intensidade moderada a vigorosa, e no aumento de tempo em comportamento sedentário. Nos Estados Unidos, observou-se um aumento no hábito de assitir à televisão (TV) e internet entre adultos durante a pandemia (Bhutani; Cooper, 2020). Resultados semelhantes foram identificados na Itália e na Espanha, tanto na participação em transmissões ao vivo, pelas redes sociais, quanto no aumento na instalação de aplicativos de programação de TV (Nielsen, 2020).

Em um período de reclusão domiciliar a população tende a adotar uma rotina sedentária, o que favoreceria a um 
aumento no ganho de peso corporal e surgimento de comorbidades associadas a maior risco cardiovascular, como obesidade, aumento da pressão arterial, intolerância à glicose, bem como transtornos psicossociais como ansiedade e depressão (Ferreira et al., 2020).

Ao passo que, tem-se que a identificação do peso de estudantes durante a pandemia da COVID-19 se faz necessária para obter o panorama do estilo de vida da comunidade, que pode ter consequências futuras. Portanto, o objeto da pesquisa baseia-se na variação ponderal de estudantes de uma escola particular de Teresina-Piauí durante isolamento social devido à pandemia Covid-19. Para levantamento do objeto citado, será realizado, por meio da aplicação de um formulário on-line, perguntas objetivas para alunos matriculados na instituição.

\section{Metodologia}

\subsection{Delineamento do estudo}

Trata-se de uma pesquisa observacional e descritiva de delineamento transversal com abordagem quantitativa.

\subsection{Local de estudo}

Fundado em 1965, o Centro Educacional Objetivo é hoje a maior instituição de ensino do Brasil, atuando no âmbito da Educação Infantil, do Ensino Fundamental e Médio e da preparação para os vestibulares. A posição foi conquistada graças ao constante aprimoramento e ao compromisso de oferecer um ensino de qualidade, capaz de formar cidadãos preparados para interagir com a comunidade e o meio ambiente em que estão inseridos. A rede de escolas conveniadas Objetivo atua em mais de 450 municípios, sendo mais de duzentos somente em São Paulo, e no Japão, totalizando mais de 430 mil alunos.

O estudo acontecerá no do Colégio Objetivo Teresina, que possui seis diferentes unidades distribuídas pela cidade, entretanto serão utilizadas no estudo apenas as que apresentam estudantes de acordo com a população delimitada. As sedes em questão estão situadas nas seguintes localizações: Unidade Centro - Avenida Frei Serafim, 1989; Unidade Leste II - Rua das Orquídeas, 988, Jóquei; Unidade Centro/Sul II - Rua Rui Barbosa, 799.

A instituição em questão possui alta infraestrutura de suporte para seus estudantes e tem como missão formar cidadãos globais íntegros, capazes de impactar o mundo, por meio de uma educação significativa. Iniciou as atividades em 2011, com foco de curso preparatório para vestibular, crescendo exponencialmente até o momento. Atualmente, dispõe de turmas desde a Educação Infantil até o preparatório para o vestibular e escolas militares.

\subsection{Período de estudo}

A coleta de dados será realizada no período de 01 de junho até 31 de julho de 2021.

\subsection{População em estudo e amostra}

Estudantes veteranos matriculados nas séries do primeiro ao terceiro ano do Ensino Médio e pré-vestibular. A instituição possui, matriculados em 2021, 1538 alunos matriculados no Ensino Médio e Pré Vestibular, dentre estes apenas 844 são veteranos.

A distribuição da amostra se encontra da seguinte forma: 213 alunos de primeiro ano, 212 alunos de segundo ano, 215 alunos de terceiro ano e 204 alunos de turmas de pré-vestibular, totalizando (N) 844 alunos.

\subsubsection{Amostra}

O estudo será realizado com 264 alunos do Ensino Médio e Pré-Vestibular do Colégio Objetivo Teresina, selecionados aleatoriamente entre os $844(\mathrm{~N})$ estudantes veteranos matriculados em 2021. 
Para determinar este número, foram usados os seguintes critérios: margem de erro (E) de 5,5\% com nível de confiança de $95 \%(Z=1,96)$ e uma variância máxima na estimativa do parâmetro da pesquisa, a variação ponderal dos estudantes, $\mathrm{P}=$ 0,50. Aplicados na seguinte fórmula, sendo (n) o tamanho da amostra para a população finita $(\mathrm{N}=844)$ :

$$
\mathrm{n}=\left(\mathrm{Z}^{2} \cdot \mathrm{P}(1-\mathrm{P}) \cdot \mathrm{N}\right) /\left(\mathrm{E}^{2} \cdot(\mathrm{N}-1)+\mathrm{Z}^{2} \cdot \mathrm{P}(1-\mathrm{P})\right)=\left(1,96^{2} \cdot 0,25 \cdot 844\right) /\left(\llbracket 0,05 \rrbracket \wedge 2.843+1,96^{2} \cdot 0,25\right)=264 \text { alunos }
$$

\subsubsection{Critérios de inclusão}

Estudantes veteranos, que estudaram na instituição durante o auge do isolamento social em 2020, atualmente matriculados no Colégio Objetivo do primeiro ao terceiro ano do Ensino Médio e do pré-vestibular que consentirem, por meio da aceitação online do TCLE, a participação na pesquisa.

\subsubsection{Critérios de exclusão}

Serão excluídos os formulários dos alunos novatos, que não eram matriculados no colégio no ano de 2020, que apresentarem dados incompletos e/ou extraviados, bem como aqueles que, por motivos operacionais (problemas no serviço de internet ou na própria plataforma), não conseguirem enviar o formulário.

\subsection{Instrumento de pesquisa}

A coleta de dados se dará por meio de um formulário on-line realizado na plataforma Google Forms ${ }^{\circledR}$ (serviço gratuito oferecido pela empresa Google para criar formulários on-line, no qual o usuário é capaz de produzir pesquisas de múltipla escolha, fazer questões discursivas, solicitar avaliações em escala numérica, entre outras opções). Tal plataforma cria automaticamente um link para ser enviado via e-mail, onde os alunos poderão clicar e serão encaminhados diretamente ao formulário. Neste, não haverá identificação garantindo o anonimato e sigilo dos dados coletados.

\subsection{Levantamento de dados}

Será entregue, via e-mail, duas vias do Termo de Consentimento Livre e Esclarecido (TCLE) para que os indivíduos possam receber esclarecimentos acerca do estudo.

A pesquisa corresponde a aplicação de um questionário on-line, que será composto por vinte e seis questões. A primeira parte do questionário será compostas por dados sociodemográficos dos participantes (idade, gênero e escolaridade). A segunda parte do questionário possui duas questões subjetivas (peso antes e após 6 meses do início do isolamento social) e vinte e duas questões que abordam sobre o estilo de vida destes estudantes.

\subsection{Análise de dados}

A análise estatística será descritiva por meio das leituras das frequências absolutas $\left(\mathrm{N}^{\circ}\right)$ e relativas $(\%)$ quando se tratar da análise de variável qualitativa e das leituras das medidas de posição (média) e de variabilidade (desvio padrão) quando se tratar de variável quantitativa. O teste de associação será o $\chi 2$ (qui-quadrado) com nível de significância $\alpha$ de 5\% (P <0,05). Os resultados estarão apresentados em formas de tabela e gráficos via Google Sheets®, serviço gratuito oferecido pela empresa Google vinculado ao Google Forms ${ }^{\circledR}$ para criar tabelas e gráficos on-line.

\subsection{Aspectos legais e éticos}

O estudo será iniciado após análise e aprovação do projeto de pesquisa pelo Comitê de Ética em Pesquisa do Centro Universitário UNINOVAFAPI. Além disso, será submetido ao referido comitê por meio da Plataforma Brasil, viabilizado pela Comissão Nacional de ética em pesquisa (CONEP), de acordo com a Resolução 466/2012 do Conselho Nacional de Saúde, a 
qual regulamenta as questões operacionais e éticas dos trabalhos científicos envolvendo seres humanos.

\subsubsection{Riscos}

Poderá haver o extravasamento de informações pessoais que será minimizado pela forma de resolução dos formulários on-line na plataforma Google Forms ${ }^{\circledR}$, visto que os voluntários não entrarão em contato com os pesquisadores diretamente. Além disso, há risco de constrangimento, reduzido pela não identificação dos participantes, assim como a não publicação de resultados individuais.

\subsubsection{Benefícios}

Esta pesquisa trará benefícios diretos para a comunidade por meio da divulgação dos resultados após sua conclusão, assim como benefícios indiretos aos próprios participantes, visto que, o ato de responder o formulário proporcionará um momento de reflexão sobre o seu padrão alimentar durante o isolamento social, além de possibilitar que despertem um olhar mais crítico e vigilante para o tema. Ademais, como o resultado dessa pesquisa será entregue para a direção da escola pesquisada, que poderá contribuir para a implementação de projetos e/ou programas de promoção da saúde destinados a pratica regular de atividades físicas durante a pandemia da COVID-19, bem como a oferta de orientações sobre alimentação de melhor qualidade.

\section{Resultados e Discussão}

Todos resultados deste trabalho seguiram as regras e normas do Comitê de Ética em Pesquisa do Centro Universitário UNINOVAFAPI e pela Comissão Nacional de Ética em Pesquisa, sendo realizado após aprovação com o número 45524721.3.0000.5210, além da realização de um termo de consentimento livre esclarecido que foi explicado para cada um dos participantes e assinado pelos mesmo após concordarem com seus termos.

Durante a pesquisa um total de 280 participantes foram coletados e responderam ao questionário disposto no Google Forms®, em que responderam questões referentes a suas idades, gênero, escolaridade, peso corporal, estado de saúde, uso de medicamentos, alimentação e rotina de atividades físicas. Para melhor distribuição dos achados, os dados serão expostos em subseções de acordo com suas finalidades.

\subsection{Perfil dos voluntários}

Os dados das medias dos perfis dos participantes está representada no quadro 1, os mesmos apresentam, na sua maioria, idade 18 anos (37.1\%), seguido de idade maior ou igual a 19 anos (36.4\%). Quanto ao gênero, mais da metade dos participantes eram mulheres $(63.2 \%)$ e com uma escolaridade com ensino médio finalizado, participando de preparatórios para o vestibular $(45 \%)$. 
Quadro 1. Distribuição das porcentagens de idade, gênero e escolaridade.

\begin{tabular}{|c|c|c|c|}
\hline \multirow{2}{*}{ Variável } & Frequência & $\%$ \\
\hline \multirow{4}{*}{ Idade } & 15 Anos & 2 & $0,7 \%$ \\
\cline { 2 - 4 } & 16 Ano & 17 & $6,1 \%$ \\
\cline { 2 - 4 } & 17 Anos & 55 & $19,6 \%$ \\
\cline { 2 - 4 } & 18 Anos & 104 & $37,1 \%$ \\
\cline { 2 - 4 } & 19 Anos ou mais & 102 & $36,4 \%$ \\
\hline \multirow{3}{*}{ Gênero } & Feminino & 177 & $63,2 \%$ \\
\cline { 2 - 4 } & Masculino & 103 & $36,8 \%$ \\
\hline \multirow{3}{*}{$\begin{array}{c}\text { Em 2020 você cursou no } \\
\text { colégio objetivo }\end{array}$} & $1^{\circ}$ Ano do ensino méd & 4 & $1,4 \%$ \\
\cline { 2 - 4 } & $2^{\circ}$ Ano do ensino méd & 61 & $21,8 \%$ \\
\cline { 2 - 4 } & $3^{\circ}$ Ano do ensino méd & 89 & $31,8 \%$ \\
\cline { 2 - 4 } & Pré vestibular & 126 & $45,0 \%$ \\
\hline
\end{tabular}

Fonte: Autores.

Avaliou-se também as condições de saúde que os participantes se encontravam, com relação a presença ou ausência de doenças crônicas e o consumo de medicamentos. Foram analisados 10 parâmetros em relação as doenças e aos medicamentos, em que as avaliavas de forma isolada ou em conjunto, ou seja, participantes com mais de um comorbidade, assim com o como a utilização de medicamentos, um ou mais de acordo com as doenças.

Com relação as enfermidades, mais da metade dos participantes afirmou não possuir nenhuma (56.1\%), a segunda maior parte (23.6\%) esta relacionada a ansiedade e depressão que é vista hoje como uma das mais comum entre os jovens, em terceiro há a categoria de outras (11.1\%), enquanto as demais variam entre 0.4-3.2\% (Figura 1).

Figura 1. Comorbidades sofridas pelos voluntários da pesquisa.

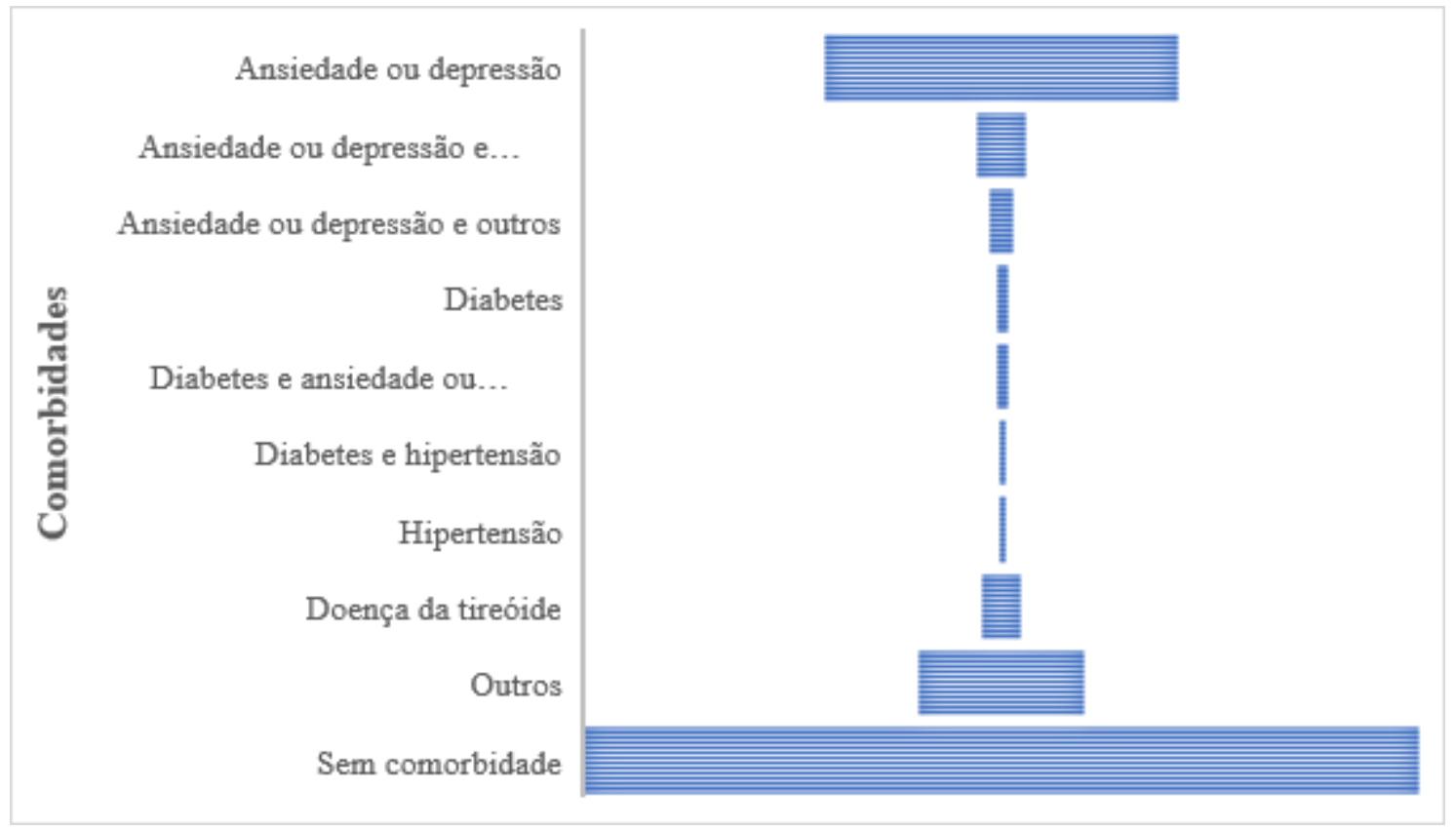

Fonte: Autores. 
Quanto os remédios e seus consumos foram proporcionais às enfermidades sofridas pelos voluntários, sendo assim a maioria não fazia uso de nenhum tipo de medicamento (71.1\%), seguido por outros medicamentos $(12.5 \%)$ e em terceiro ansiolítico ou antidepressivos (11.1\%), os demais tipos ficaram com distribuição de consumo entre 0.4-2.5\% (Figure 2).

Figura 2. Medicamentos consumidos pelos voluntários da pesquisa.

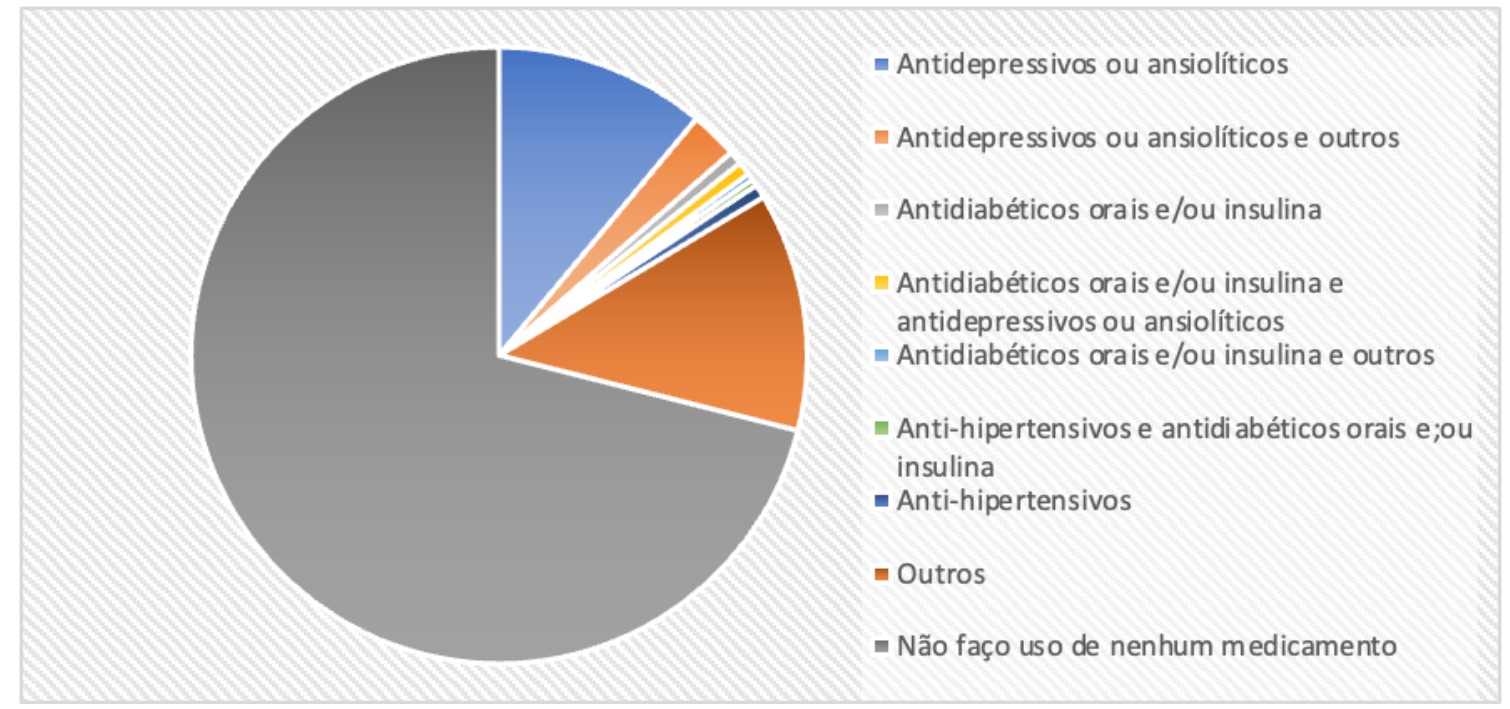

Fonte: Autores.

\subsection{Variação do peso e hábitos alimentares nos períodos anterior e seis meses após inicio da pandemia}

Os 280 participantes apresentaram uma media de peso corporal de $62.67 \pm 12.57 \mathrm{~kg}$ no início da pandemia, sendo os pesos de 50,62 e $70 \mathrm{~kg}$ os mais apresentados, 14\% para cada. Após o período de seis meses, o peso médio dos participantes apresentou um leve aumento, indo para $66.53 \pm 13.59 \mathrm{~kg}$, com o peso mais comum entre os participantes de $68 \mathrm{~kg}$ (Figura 3).

A variação média do peso durante esse período foi de $3.77 \pm 5.11$, o alto desvio padrão nessa medida se dá pela diversidade entre os 280 voluntários que apresentaram tanto ganho quanto perda de peso, tal feito fez com que houvesse uma grande variação dos valores.

Figura 3. Gráfico de variação dos pesos antes e após 6 meses de isolamento.

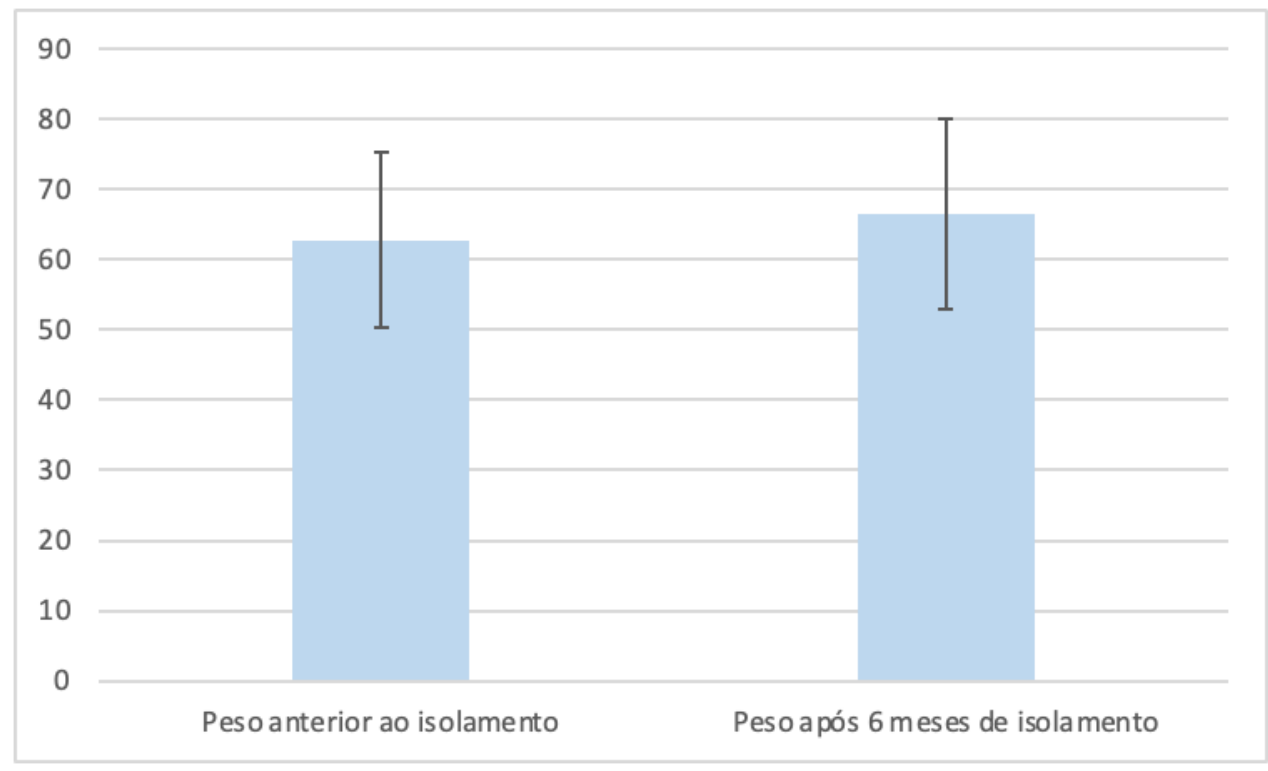

Fonte: Autores. 
Os aspectos alimentares também foram analisados, cerca de $89.6 \%$ dos participantes assinalaram que seus hábitos alimentares não sofreram alteração durante o período de seis meses após o início da pandemia, enquanto os $10.4 \%$ restantes relataram que sim, essas mudanças podem estar relacionadas ao número de refeições feitas e a suas origens.

Inicialmente a quantidade de refeições diárias feitas pelos participantes (Figura 4), que antes da pandemia eram na sua maioria de 3-4 (50.4\%) passou a apresentar uma grande variabilidade, apesar do maior percentual permanecer na mesma faixa de quantidade, com $28.6 \%$ dos participantes ingerindo de 3-4 refeições diárias, alguns dos participantes relataram fazer de nove a mais refeições por dia $(10.4 \%)$.

Figura 4. Distribuição da quantidade de refeições diárias antes e 6 meses após isolamento.

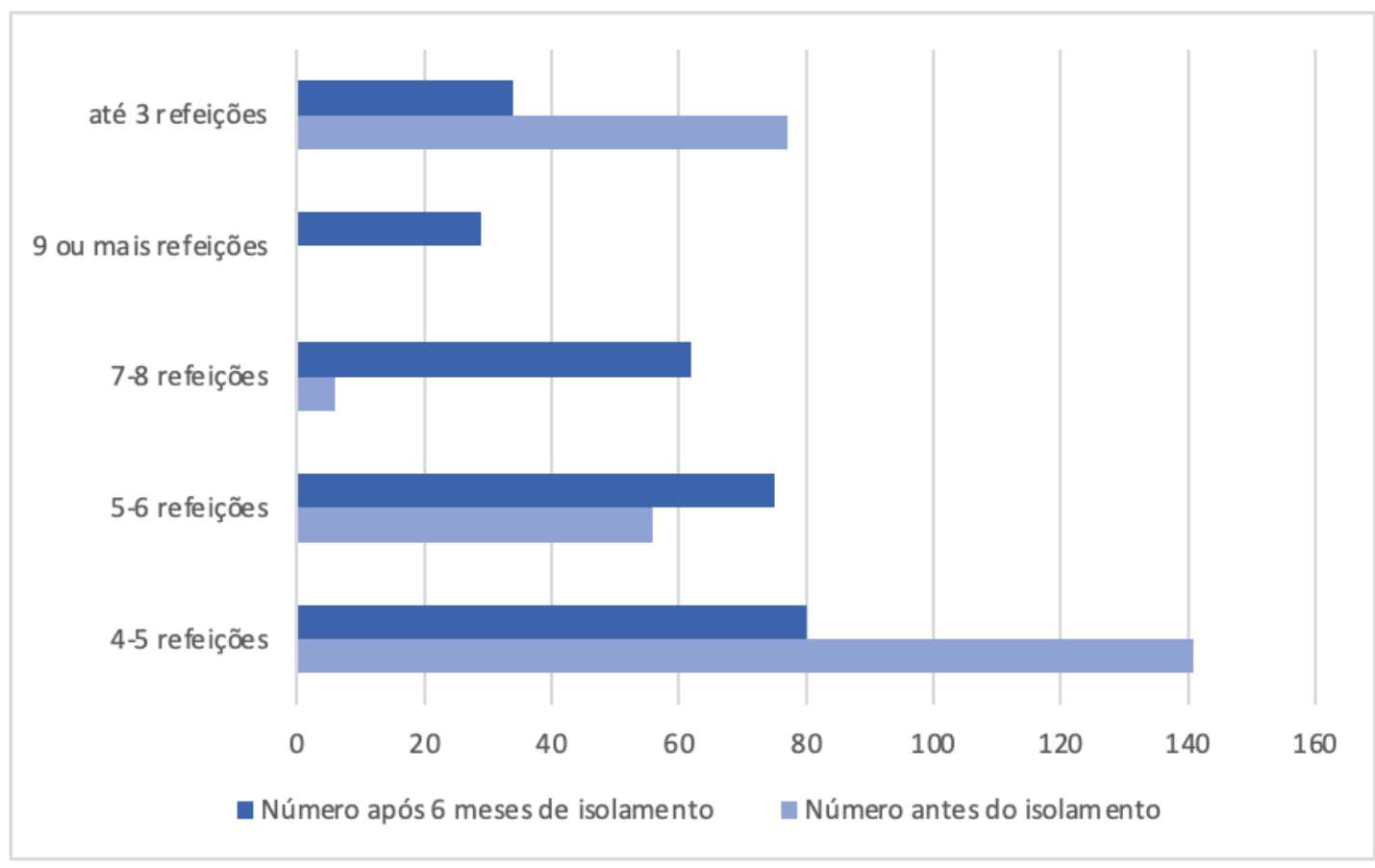

Fonte: Autores.

Dentro dos aspectos alimentares, o tipo consumido pelos participantes também foi avaliado. Em questão principal a presença de alimentos caseiros, fast foods, delivery, consumo de enlatado, fritura e refrigerante e de produtos industrializados e pobres em fibras. No período anterior a pandemia $92.1 \%$ dos participantes realizavam principalmente refeições caseiros, enquanto $3.9 \%$ eram fast food e $3.9 \%$ delivery, após o início da pandemia esse valor passou a ser $67.9 \%$, $8.2 \%$ e $23.9 \%$, respectivamente, mostram um aumento de $612.8 \%$ no consumo de delivery.

O consumo semanal de cada um dos tipos de alimento também foi analisado, antes da pandemia (Figura 5) o consumo diário era majoritariamente de comida caseiras (81.4\%), enquanto delivery $(73.3 \%)$ e fast food (83.64\%) eram consumidos de 1 a $3 x$ por semana ou então nunca eram consumidos, $27.5 \%$ e $15.4 \%$, respectivamente.

Ainda foi indagado aos participantes o aumento do consumo de comidas caseiras e de delivery durante a pandemia, cerca de $62.5 \%$ afirmaram que aumentaram o consumo de refeições caseiras, em contra partida $87.1 \%$ de todos os entrevistados também afirmaram que o pedido por delivery cresceu durante o mesmo período, importante ressaltar que essas populações podem se intercalar e estar presente em uma não excluí a possibilidade do voluntário estar na outra. 
Research, Society and Development, v. 11, n. 3, e18211326518, 2022

(CC BY 4.0) | ISSN 2525-3409 | DOI: http://dx.doi.org/10.33448/rsd-v11i3.26518

Figura 5. Consumo dos tipos de alimentos por frequência semanal antes do isolamento.

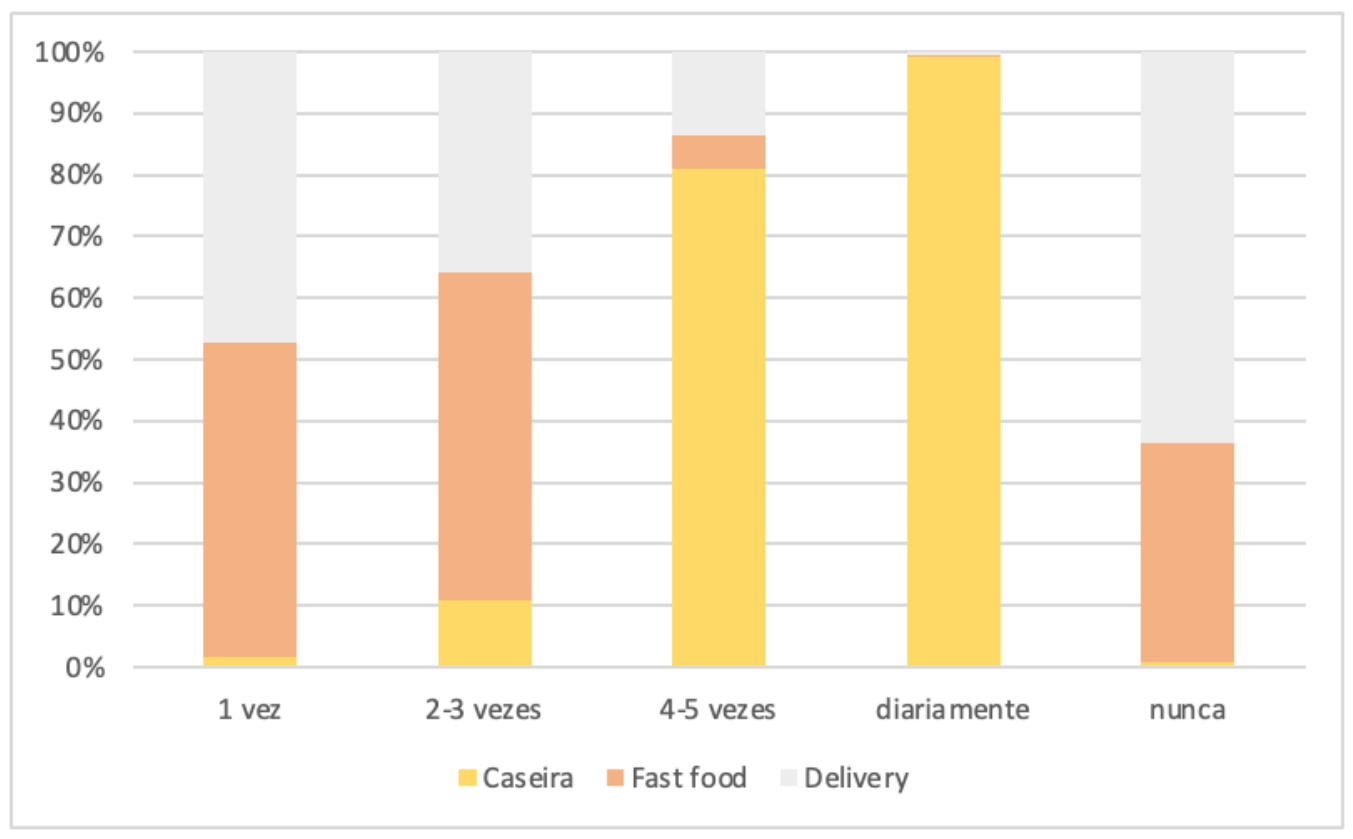

Fonte: Autores.

A análise dos seus consumos seis meses após pandemia observa-se uma mudança de cenário, como dito anteriormente 87.1\% afirmou ter aumentado o consumo de delivery, esse fato é refletido na Figura 6, em que essa categoria de alimento passa a ter mais expressão quando comparado ao gráfico anterior, em que seu consumo chega a ser feito de forma diária por alguns participantes, assim como o de fast food.

Figura 6. Consumo dos tipos de alimentos por frequência semanal após 6 meses de isolamento.

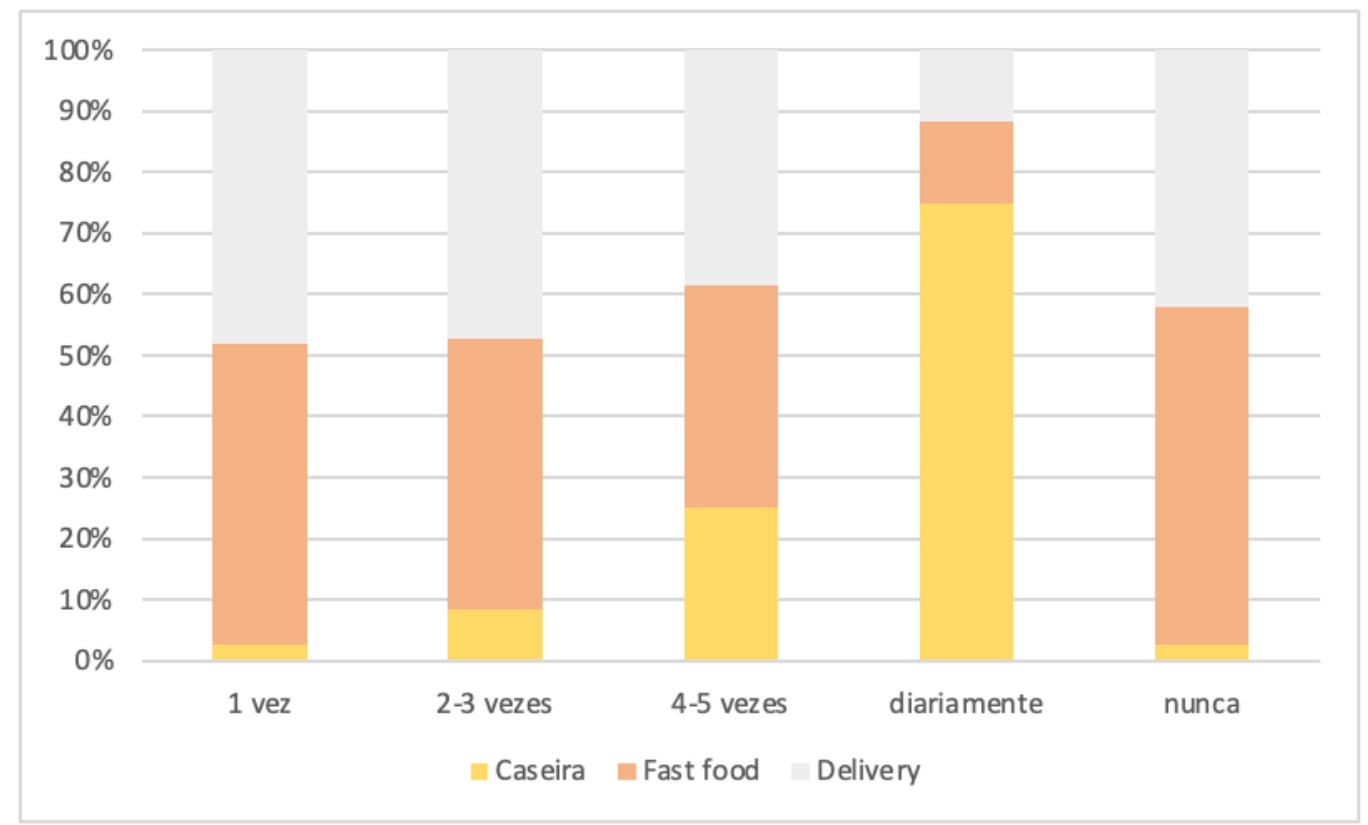

Fonte: Autores.

Apesar desse aumento de delivery e fast food para até 5 vezes por semana, alimentos caseiros continuam sendo os mais consumidos diariamente, apesar de terem apresentado um leve aumento na barra de nunca consumidos, o mesmo 
permanece apenas com 1 participante assinalando nunca, tenho essa variação da proporção devido às demais categorias.

Com relação ao consumo de enlatados, refrigerantes e frituras, $81.8 \%$ dos participantes passaram a ingerir tais alimentos após o inicio da pandemia, juntamente com isso $84.3 \%$ dos 280 voluntários afirmam que aumentaram o consumo de produtos industrializados e pobres em fibra em comparação a período anterior a pandemia.

O aumento de consumo de enlatados, frituras e refrigerante não aumentou apenas na quantidade, mas também na sua frequência. Como observado no gráfico abaixo (Figura 7) esses alimentos que antes eram consumidos normalmente até 3x por semana, passaram para a ter uma maior presença no prato dos participantes, chegando a alcançar um consumo diário de $28.9 \%$.

Figura 7. Consumo de enlatados, refrigerante e frituras antes e após 6 meses de isolamento.

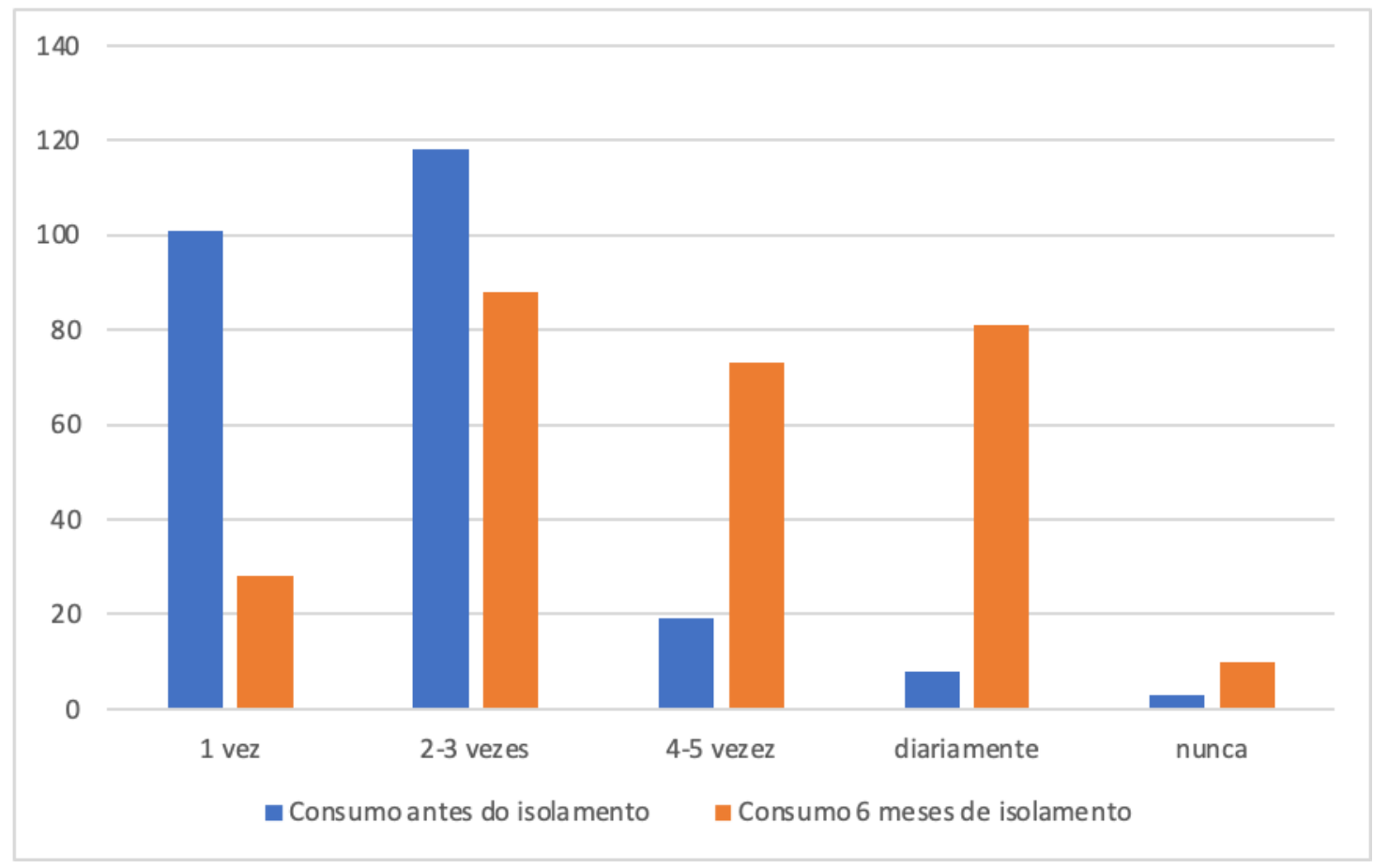

Fonte: Autores.

Por último foi analisado os motivos que levaram a uma não manutenção da alimentação adequada durante a pandemia, os fatores variaram entre ansiedade, compulsão, medo, estresse, desânimo e outros, de forma isolada e em conjunto. Apesar das variadas opções $10.4 \%$ dos participantes não responderam à pergunta, $10 \%$ dos que responderam afirmaram que a ansiedade foi o principal causador do consumo de alimentos, seguido de compulsão e ansiedade (7.5\%) e em terceiro a combinação dos cinco fatores $(7.1 \%)$.

Outros motivos como compulsão, ansiedade e outros e estresse, compulsão e ansiedade tivera uma porcentagem de $6.1 \%$, as demais combinações apresentaram valores que ficaram entre 5.4-0.4\%.

\subsection{Realização de atividade física e suas modalidades}

A manutenção da rotina de atividade físicas ficou afetada para 78.9\% dos participantes, que não conseguiram manter a mesma que tinham antes do isolamento. A frequência com a qual praticavam as atividades antes desse período também foi analisado (Figura 8), em que a maioria assinalou realizar as atividades 2 a 3 vezes por semana (38.2\%). 
Figura 8. Frequência da pratica de atividade física por parte dos participantes.

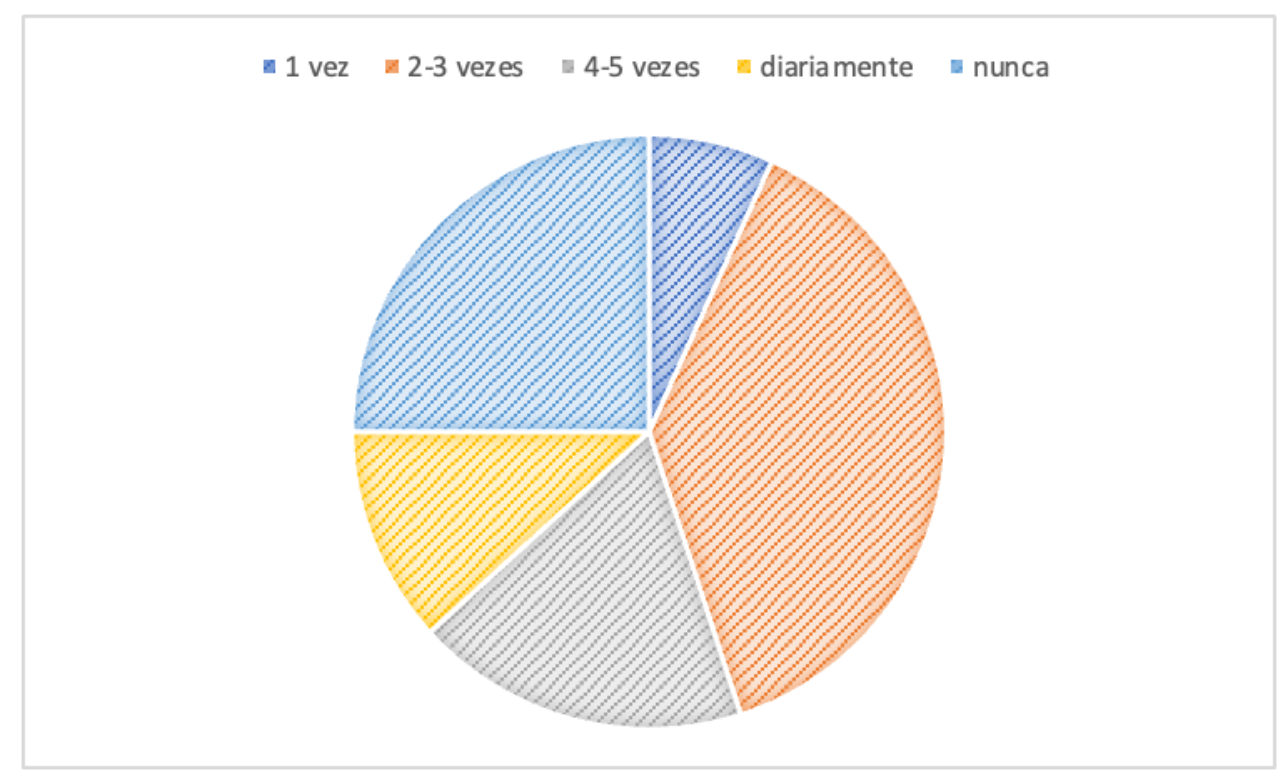

Fonte: Autores.

Os motivos pelos quais não foi possível manter a rotina de treino foram avaliadas pelos pesquisadores, as opções para tal variavam entre o ambiente de exercício ser em academia ou box fechado, ansiedade, desânimo, estresse, medo e outro, além da combinação entre os fatores. Como fator principal foi assinalado a opção outros (18.9\%), seguido de desânimo (17.1\%), a combinação entre desânimo e academia ou box fechado (10.7\%) e o ambiente de exercício está fechado foi o quarto fator que mais influenciou a quebra da rotina, os demais fatores tiveram uma porcentagem entre 7.1-0.4\%.

Dada a alta prevalência de complicações graves do COVID-19 com ênfase às associadas à obesidade, compreender o impacto do bloqueio no ganho de peso prejudicial à saúde e na obesidade é de importância significativa para a saúde pública (Bhutani; Cooper, 2020; Zachary et al., 2020).

Alguns autores relatam o aumento da ingestão de alimentos em resposta à visão e ao olfato durante a quarentena pode ser considerado um componente das variáveis psicológicas impulsividade e desinibição. A impulsividade é uma reação apressada a um estímulo externo sem preocupação com as consequências negativas e tem sido associada à compulsão alimentar (Krznarić et al., 2020; Zachary et al., 2020; Liu et al., 2020; Haraj et al., 2021).

Problemas relacionados a saúde mental podem estar relacionados a compulsão alimentar em jovens, nesse sentido um estudo apontou que o amplo escopo e disseminação do COVID-19 poderia levar a uma verdadeira crise de saúde mental, especialmente em países com alta carga de casos (Rajkumar, 2020). No presente a maioria dos participantes relatou não possui nenhum problema de saúde mental (56.1\%), em contrapartida a segunda maior parte (23.6\%) relatou possuir tais problemas principalmente relacionados a ansiedade e depressão

Para Rundle et al (2020) o fechamento de escolas criam ambiente alimentar e desafios para a realização de atividade física em crianças e adolescentes. A interrupção de um estado de inibição em que pode ocorrer perda de controle sobre a alimentação também está relacionada ao ganho de peso, dessa forma existem relatos de autores que descreveram que durante a quarentena, aqueles que ganharam peso simplesmente comeram porque havia comida disponível, não necessariamente por causa de sinais internos de fome.

Yu et al (2021) aborda a idade como um dos fatores mais importantes na taxa de hospitalização do COVID-19, com cerca de 70\% dos casos hospitalizados com idade superior a 45 anos e obesidade, como condição de base mais comum para pacientes com COVID-19, dessa forma tal condição pode transferir o risco de complicações pelo Sars-Cov-2 para idades mais 
jovens.

Na presente pesquisa ouve o aumento de peso nos participantes corroborando com o estudo de Mulugeta; Desalegn; Solomon (2021) em que os autores observaram que 40,6\% dos homens e 46,1\% das mulheres ganharam peso. Destes, $26,8 \%$ dos homens e $29,5 \%$ das mulheres ganharam peso clinicamente significativo de mais de $5 \%$ do peso basal.

Embora o ganho de peso durante a pandemia de COVID-19 entre adultos ainda não tenha sido relatado, um grande estudo entre jovens chineses encontrou um aumento significativo no índice de massa corporal, bem como nas taxas de sobrepeso e obesidade (Jia et al., 2020).

No estudo realizado por Rundle et al (2020) foi demostrado também que embora não medidos diretamente, adolescentes que mantiveram ou perderam peso durante o isolamento social provavelmente praticavam contenção alimentar. A restrição alimentar é definida como a intenção de restringir a ingestão de alimentos para controlar o peso e a forma corporal e foi mostrado para prever a perda de peso a longo prazo, deve-se encorajar a prática de restrição alimentar alimentos pobres em nutrientes como fast-foods e encorajar a alimentação saudável durante a quarentena (JIA et al., 2020; Bhutani; Cooper, 2020).

A pesquisa de $\mathrm{Yu}$ et al (2020) apoia a descoberta de que as taxas de obesidade aumentaram na pandemia. Uma pesquisa de março da American Psychological Association descobriu que $42 \%$ dos americanos disseram que ganharam mais peso do que o pretendido, $13 \mathrm{~kg}$ em média, sendo um valor mais elevado que o apresentado neste estudo.

Um estudo recente que reuniu dados de registros de saúde pediátrica encontrou uma tendência de ganho de peso em crianças, especialmente entre 5 e 11 anos. Nessa faixa etária, o sobrepeso ou obesidade aumentou de 36,2\% para 45,7\% durante a pandemia (Wen; Zhu; Ji, 2021).

Fernandez-rio et al (2020) relataram que cerca de $40 \%$ da população diminuirá a ingestão de alimentos em resposta ao estresse, $40 \%$ relatam aumento da ingestão de alimentos e $20 \%$ relatam nenhuma mudança. Assim, embora os níveis de estresse estivessem no nível que indicava estresse moderado, é mais do que provável que apenas entre aqueles que usam os alimentos como mecanismo de enfrentamento isso afetou de forma deletéria o peso. $\mathrm{O}$ estudo atual não avaliou diferenças na resposta ao estresse (Fernandez-Rio et al., 2020).

A ligação entre lanches após o jantar e ganho de peso é bem suportada na literatura. Dados emergentes revelaram que uma alta porcentagem de tecido adiposo é regulada diurnamente e que as calorias consumidas no final do dia têm uma maior propensão a serem armazenadas no tecido adiposo (Zachary et al., 2020).

A disseminação da regulação alimentar e de estratégias de enfrentamento saudáveis é de importância crítica durante a pandemia de COVID-19 para prevenir o ganho de peso e padrões alimentares pouco saudáveis. No contexto atual, a telessaúde, a saúde móvel (por exemplo, aplicativos para smartphones) e as intervenções ecológicas momentâneas voltadas para a redução da alimentação para lidar com a pandemia são garantidas (Cooper, 2020).

Além disso, as mensagens da mídia de massa que combatem as estratégias de alimentação pouco saudáveis podem ser relevantes para atenuar qualquer impacto adverso da pandemia sobre a obesidade, sendo utilizada como as estratégias de manutenção do peso, como a autopesagem diária e o automonitoramento, podem ser úteis para a prevenção do ganho de peso durante a pandemia de COVID-19 (Wen; Zhu; Ji, 2021; Yu et al., 2021).

Neste sentido Yu et al (2020) enfatizaram que estratégias inovadoras, robustas e altamente adaptáveis devem ser adotadas para aumentar a atividade física, reduzir o tempo sedentário e promover dietas saudáveis, para minimizar o impacto adverso do fechamento de escolas.

\section{Conclusão}

Os dados da pesquisa sugerem que a pandemia ocasionada pelo Sars-Cov-2 foi associado a maiores aumentos no peso corporal depois do que antes da pandemia. A disseminação da regulação alimentar e de estratégias de enfrentamento saudáveis 
é de importância crítica durante a pandemia de COVID-19 para prevenir o ganho de peso e padrões alimentares pouco saudáveis.

No contexto atual, a telessaúde, a saúde móvel (por exemplo, aplicativos para smartphones) e as intervenções ecológicas momentâneas voltadas para a redução da alimentação para lidar com a pandemia são garantidas. Além disso, as mensagens da mídia de massa que combatem as estratégias de alimentação pouco saudáveis podem ser relevantes para atenuar qualquer impacto adverso da pandemia.

Dado o surgimento do COVID-19, e o isolamento social com fechamento de escolas seria útil conduzir um estudo adicional para identificar quaisquer mudanças adicionais no comportamento alimentar de pacientes afetados por sobrepeso/obesidade neste momento, a fim de orientar o desenvolvimento de medicina personalizada, e intervenções psicológicas, psicossociais.

\section{Referências}

Baysun, Ş., \& Akar, M. N. (2020). Weight Gain in Children During the Covid-19 Quarantine Period. Journal of paediatrics and child health.

Bhutani, S., Cooper, J. A., \& Vandellen, M. R. (2021). Self-reported Changes in Energy Balance Behaviors during COVID-19-related Home Confinement: A Cross-sectional Study. American Journal of Health Behavior, 45(4), 756-770.

Brasil, M. S. Secretaria de Atenção à Saúde. Departamento de Atenção Básica. Guia alimentar para a população brasileira. 2. ed. Brasília: Ministério da Saúde, 2014 .

Chan, J. F. W., Yuan, S., Kok, K. H., To, K. K. W., Chu, H., Yang, J., Yuen, K. Y. (2020). A familial cluster of pneumonia associated with the 2019 novel coronavirus indicating person-to-person transmission: a study of a family cluster. The lancet, 395(10223), 514-523.

Gorbalenya, A. E., Baker, S. C., Baric, R., Groot, R. J. D., Drosten, C., Gulyaeva, A. A., Ziebuhr, J. (2020). Severe acute respiratory syndrome-related coronavirus: The species and its viruses-a statement of the Coronavirus Study Group.

Fernandez-Rio, J., Cecchini, J. A., Mendez-Gimenez, A., \& Carriedo, A. (2020). Weight changes during the COVID-19 home confinement. Effects on psychosocial variables. Obesity Research \& Clinical Practice, 14(4), 383-385.

Ferreira, M. J., Irigoyen, M. C., Consolim-Colombo, F., Saraiva, J. F. K., \& Angelis, K. D. (2020). Physically active lifestyle as an approach to confronting COVID-19. Arquivos Brasileiros de Cardiologia, 114(4), 601-602.

Haraj, N. E., El Aziz, S., Chadli, A., Dafir, A., Mjabber, A., Aissaoui, O., ... \& Afif, M. H. (2021). Nutritional status assessment in patients with Covid-19 after discharge from the intensive care unit. Clinical Nutrition ESPEN, 41, 423-428.

Jia, P., Zhang, L., Yu, W., Yu, B., Liu, M., Zhang, D., \& Yang, S. (2021). Impact of COVID-19 lockdown on activity patterns and weight status among youths in China: the COVID-19 Impact on Lifestyle Change Survey (COINLICS). International Journal of Obesity, 45(3), $695-699$.

Junior, L. C. L. (2020). Alimentação saudável e exercícios físicos em meio à pandemia da COVID-19. Boletim de conjuntura (boca), 3(9), 33-41.

Júnior, P. G. F., Paiano, R., \& dos Santos Costa, A. (2020). Isolamento social: consequências físicas e mentais da inatividade física em crianças e adolescentes. Revista Brasileira de Atividade Física \& Saúde, 25, 1-2.

Kananen, L., Eriksdotter, M., Boström, A. M., Kivipelto, M., Annetorp, M., Metzner, C., ... \& Cederholm, T. (2021). Body mass index and Mini Nutritional Assessment-Short Form as predictors of in-geriatric hospital mortality in older adults with COVID-19. Clinical Nutrition.

Krznarić, Ž., Bender, D. V., Laviano, A., Cuerda, C., Landi, F., Monteiro, R., ... \& Barazzoni, R. (2020). A simple remote nutritional screening tool and practical guidance for nutritional care in primary practice during the COVID-19 pandemic. Clinical nutrition, 39(7), $1983-1987$.

Liu, G., Zhang, S., Mao, Z., Wang, W., \& Hu, H. (2020). Clinical significance of nutritional risk screening for older adult patients with COVID-19. European journal of clinical nutrition, 74(6), 876-883.

Machida, M., Nakamura, I., Saito, R., Nakaya, T., Hanibuchi, T., Takamiya, T., ... \& Inoue, S. (2020). Adoption of personal protective measures by ordinary citizens during the COVID-19 outbreak in Japan. International journal of infectious diseases, 94, 139-144.

Malta, D. C., Szwarcwald, C. L., Barros, M. B. D. A., Gomes, C. S., Machado, Í. E., Souza Júnior, P. R. B. D., ... \& Gracie, R. (2020). A pandemia da COVID-19 e as mudanças no estilo de vida dos brasileiros adultos: um estudo transversal, 2020. Epidemiologia e Serviços de Saúde, 29.

McAtamney, K., Mantzios, M., Egan, H., \& Wallis, D. J. (2021). Emotional eating during COVID-19 in the United Kingdom: Exploring the roles of alexithymia and emotion dysregulation. Appetite, 161, 105120.

Mulugeta, W., Desalegn, H., \& Solomon, S. (2021). Impact of the COVID-19 pandemic lockdown on weight status and factors associated with weight gain among adults in Massachusetts. Clinical obesity, 11(4), e12453.

Nielsen, B. F., Sneppen, K., Simonsen, L., \& Mathiesen, J. (2020). Social network heterogeneity is essential for contact tracing. medRxiv. 
Qiu, Y., Chen, X., \& Shi, W. (2020). Impacts of social and economic factors on the transmission of coronavirus disease 2019 (COVID-19) in China. Journal of Population Economics, 33(4), 1127-1172.

Rajkumar, R. P. (2020). COVID-19 and mental health: A review of the existing literature. Asian journal of psychiatry, $52,102066$.

Ribeiro, K. D. D. S., Garcia, L. R. S., Dametto, J. F. D. S., Assunção, D. G. F., \& Maciel, B. L. L. (2020). COVID-19 and nutrition: the need for initiatives to promote healthy eating and prevent obesity in childhood. Childhood obesity, 16(4), 235-237.

Rundle, A. G., Park, Y., Herbstman, J. B., Kinsey, E. W., \& Wang, Y. C. (2020). COVID-19 related school closings and risk of weight gain among children. Obesity (Silver Spring, Md.), 28(6), 1008.

Santos Rodrigues, J. V., \& de Almeida Lins, A. C. A. (2020). Possíveis impactos causados pela pandemia da COVID-19 na saúde mental de crianças e o papel dos pais neste cenário. Research, Society and Development, 9(8), e793986533-e793986533.

Sidor, A., \& Rzymski, P. (2020). Dietary choices and habits during COVID-19 lockdown: experience from Poland. Nutrients, $12(6), 1657$.

Silva, L. L. S. D., Lima, A. F. R., Polli, D. A., Razia, P. F. S., Pavão, L. F. A., Cavalcanti, M. A. F. D. H., \& Toscano, C. M. (2020). Medidas de distanciamento social para o enfrentamento da COVID-19 no Brasil: caracterização e análise epidemiológica por estado. Cadernos de Saúde Pública, 36.

Szwarcwald, C. L., Souza Júnior, P. R. B. D., Malta, D. C., Barros, M. B. D. A., Magalhães, M. D. A. F. M., Xavier, D. R., ... \& Pina, M. D. F. D. (2020). Adesão às medidas de restrição de contato físico e disseminação da COVID-19 no Brasil. Epidemiologia e Serviços de Saúde, 29 , e2020432.

WHO, W. H. O. Pneumonia of unknown cause - China.2020. https://www.who.int/csr/don/05-january-2020-pneumonia-of-unkown-cause-china/en/

Wen, J., Zhu, L., \& Ji, C. (2021). Changes in weight and height among Chinese preschool children during COVID-19 school closures. International Journal of Obesity, 45(10), 2269-2273.

$\mathrm{Xu}$, Y. (2020). Unveiling the origin and transmission of 2019-nCoV. Trends in microbiology, 28(4), 239-240.

Yu, W., Rohli, K. E., Yang, S., \& Jia, P. (2021). Impact of obesity on COVID-19 patients. Journal of Diabetes and its Complications, $35(3), 107817$.

Zachary, Z., Brianna, F., Brianna, L., Garrett, P., Jade, W., Alyssa, D., \& Mikayla, K. (2020). Self-quarantine and weight gain related risk factors during the COVID-19 pandemic. Obesity research \& clinical practice, 14(3), 210-216.

Zheng, R., Xu, Y., Wang, W., Ning, G., \& Bi, Y. (2020). Spatial transmission of COVID-19 via public and private transportation in China. Travel medicine and infectious disease, 34, 101626.

Zhu, N., Zhang, D., Wang, W., Li, X., Yang, B., Song, J., ... \& Tan, W. (2020). A novel coronavirus from patients with pneumonia in China, 2019. New England journal of medicine. 